\title{
Real time moire interferometry using a Fabry-Perot cavity with a phase conjugate mirror
}

\author{
Guy Indebetouw and Kanwai Peter Lo
}

\begin{abstract}
We describe a self-referencing interferometer in which the wavefront and its complex conjugate are generated in a Fabry-Perot cavity having one phase conjugate mirror (PCM). The finite response time of the PCM allows one to display momentarily the moiré between old and new interferograms after a sudden change has affected the input wavefront. Results using an externally pumped $\mathrm{BaTiO}_{3}$ crystal as a $\mathrm{PCM}$ are analyzed.
\end{abstract}

\section{Introduction}

Self-referencing interferometers display the interference of a wavefront and a modified version of itself (e.g., translated, magnified, folded, inverted ... etc.). These interferometers are sensitive, do not require an external reference wave, and have a fringe contrast independent of the spatial input intensity variations.

Of particular interest are the interferometers which display an interferogram of a wavefront and its complex conjugate. They have double the sensitivity of equivalent conventional interferometers. It is always possible to produce the phase conjugate wavefront by reconstruction of conventional holograms ${ }^{1}$ but this precludes real-time operation. The prospect of being able to generate the conjugate wave dynamically, however, opens up a whole new range of possibilities. Indeed, a number of real-time self-referencing interferometers using nonlinear wave mixing to generate the phase conjugate wave have been proposed ${ }^{2}$ and demonstrated $^{3,4}$ several years ago. These interferometers are of the two-wave type (Michelson or MachZehnder). More recently, phase conjugate mirrors (PCM) using photorefractive materials have been introduced in one ${ }^{5}$ or both arms $s^{6,7}$ of a Michelson interferometer to produce devices sensitive to the temporal changes of a wavefront. Highly sensitive photorefractive materials are ideal for this type of application. They make low power devices possible and their relatively slow response can often be used to advantage. Other wave mixing geometries have also been proposed; for example, to generate the wavefront and its complex conjugate colinearly in a nonlinear material. ${ }^{8}$

It is well known that in an optical cavity having one phase conjugate mirror, two fields coexist, the input field and its complex conjugate. ${ }^{9}$ The first field re-

The authors are with Virginia Polytechnic Institute \& State University, Physics Department, Blacksburg, Virginia 24061-0435.

Received 10 January 1989.

0003-6935/89/183893-04\$2.00/0.

(C) 1989 Optical Society of America. sults from the superposition of beams which have been phase conjugated an even number of times. The other one results from the superposition of beams conjugated an odd number of times. Such phase conjugate cavities, either of the linear or of the ring type, are thus natural self-referencing interferometers.

In this paper, we discuss the use of a Fabry-Perot cavity with an externally pumped PCM as a self-referencing interferometer. The device has several advantages. The steady-state field in the cavity is the desired interferogram of a wavefront and its complex conjugate. Alignment of the cavity is not critical and background fringes can be easily introduced. The finite response time of the PCM can be exploited to display momentarilly the beats (moiré) between two interferograms, providing a means of measuring, in real time, sudden changes occuring in the input wavefront.

As mentioned in Ref. 2, this type of interferometer is self-referencing in a somewhat restricted sense. It does indeed produce an interferogram of the wavefront and its complex conjugate and the fringe contrast is unsensitive to the input intensity. However, due to the fact that the PCM is externally pumped, it remains sensitive to the relative phase of the pumps and the signal beams. A self-pumped PCM would not suffer this drawback. However, it would lack an interesting additional degree of freedom. The possibility of varying the gain of the PCM, especially near the threshold for self-oscillation, was found to greatly influence the dynamic properties of the interferometer.

The theory is outlined in the next section. The setup is then described in Sec. III together with some experimental results.

\section{Theory}

A schematic of the Fabry-Perot cavity is shown in Fig. 1. The mirror $M$ and the beam splitter $B S$ are lossless and their Fresnel reflection and transmission coefficients satisfy the Stokes relationships:

$$
\begin{aligned}
r^{*} t+r^{\prime} t^{*} & =0, \\
t^{*} t^{\prime} & =1-|r|^{2} .
\end{aligned}
$$




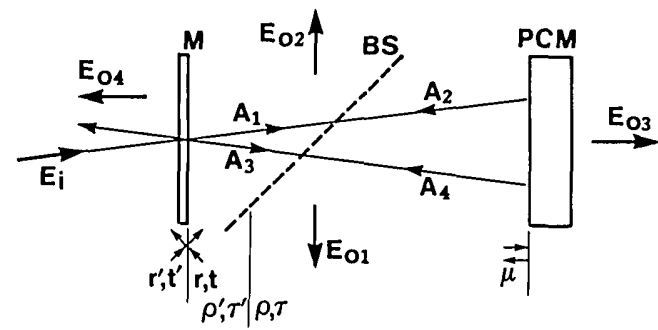

Fig. 1. Fabry-Perot cavity with a phase conjugate mirror (PCM). The output of the self-referencing interferometer is $E_{O 1}$.

Similar relationships hold for the beam splitter with $\rho, \tau$ replacing $r, t$. For simplicity, the phase conjugate mirror is characterized by a single reflectivity coefficient $\mu$ which may be, however, complex or larger than unity.

There are four possible output posts shown as $E_{O 1}$ to $E_{O 4}$ in Fig. 1. The output of the self-referencing interferometer considered here is $E_{01}$. It is a superposition of the fields $A_{2}$ and $A_{4}$ in the cavity. $A_{2}$ (respectively $A_{4}$ ) results from the input having been phase conjugated an odd (even) number of times and is thus proportional to $E_{i}^{*}\left(E_{i}\right)$. The steady state output is given by

$$
E_{O 1}=\rho \tau^{\prime *} \mu\left(1-|\alpha|^{2}\right)^{-1}\left(t^{\prime} \alpha^{*} E_{i}+t^{* *} E_{i}^{*}\right),
$$

where

$$
\alpha=r \tau \tau^{\prime *} \mu=r \mu\left(1-|\rho|^{2}\right)
$$

is the feedback parameter of the cavity which can be adjusted by varying the gain of the PCM. The threshold for self-oscillation is $|\alpha|=1$. Note that the PCM reflectivity $\mu$ appears in both terms of the output. This means that a sudden change in the input will only appear in the output gradually, with a time constant equal to the response time of the PCM.

It is worthwhile to briefly mention the outputs at the other posts. Output $E_{O 2}$ is given by

$$
E_{O 2}=\rho^{\prime}\left(1-|\alpha|^{2}\right)^{-1}\left(t^{\prime} E_{i}+t^{\prime *} \alpha E_{i}^{*}\right) .
$$

The first term is the direct input and will therefore carry instantly to the output any sudden changes occuring in the input. Output $E_{O 3}$ has similar properties. It may, however, show additional phase distorsions due to the transmission through the PCM. Output $E_{O 4}$, without the beam splitter $B S$, is given by

$$
E_{O 4}=\left(1-|\mu r|^{2}\right)^{-1}\left[\left(1-|\mu|^{2}\right) r^{\prime} E_{i}+\left(1-|r|^{2}\right) \mu E_{i}^{*}\right] .
$$

This output has been studied to demonstrate a remarkable property of PCM's: An ideal PCM $(\mu \equiv 1)$ will exactly compensate for the scattering of the Fabry-Perot cavity and produce an exact phase conjugate output $E_{O 4} \equiv E_{i}^{*} \cdot 10,11$

The dynamics of a Fabry-Perot with PCM has been studied in the case of a PCM having an instantaneous response. ${ }^{12}$ The time constants involved in the process are then the cavity lifetime (or filling time), which depends on the finesse and on the gain, the cavity round trip time, and the coherence time of the radiation.

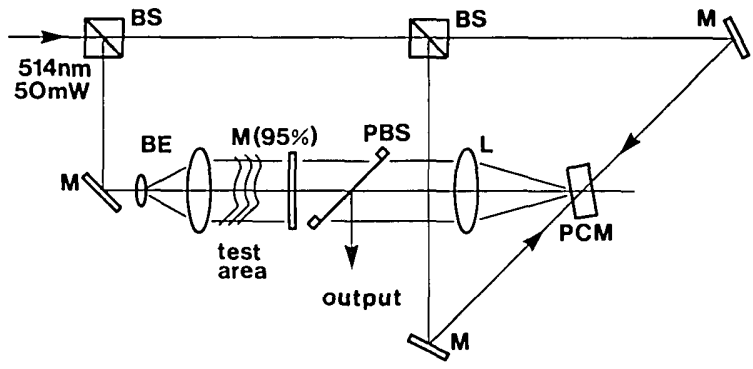

Fig. 2. Setup of the self-referencing interferometer. ( $B S$ beam splitter, $M$ mirrors, $B E$ beam expander, $P B S$ pellicle beam splitter, $P C M$ phase conjugate mirror).

In our experiments, we used a relatively slow medium $\left(\mathrm{BaTiO}_{3}\right)$ for the PCM. Furthermore, it is the finite response time of the PCM which makes it possible to display the moiré between old and new interferograms when a sudden change occurs in the input. If the response time $\tau$ of the PCM is much larger than the filling time, we may assume a quasi-steady-state or adiabatic condition in which the cavity is instantly filled after each abrupt change of input. The output will then evolve with the same time constant $\tau$ as the response time of the PCM. Of course, if the input changes continuously, these changes must be slow compared with the PCM response time in order for the output to follow and display the changing interferogram.

If the input changes abruptly from $E_{i 1}$ to $E_{i 2}$, the old interferogram stored in the PCM will decay while the new one builds up in the cavity with a time constant $\tau$. Near threshold $(|\alpha| \lesssim 1)$, the output has the approximate form

$$
E_{O 1}(t) \propto\left(\alpha^{*} E_{i 1}+E_{i 1}^{*}\right) e^{-t / \tau}+\left(\alpha^{*} E_{i 2}+E_{i 2}^{*}\right)\left(1-e^{-t / \tau}\right),
$$

where $\alpha \approx \exp (i \epsilon), \epsilon=\arg \mu \mathrm{r}$. As an example, a phase input changing suddenly from $\exp \left(i \phi_{1}\right)$ to $\exp \left(i \phi_{2}\right)$ produces an output

$$
E_{O 1}(t) \propto \cos \left(\phi_{1}+\epsilon / 2\right) e^{-t / \tau}+\cos \left(\phi_{2}+\epsilon / 2\right)\left(1-e^{-t / \tau}\right) .
$$

During a lapse of time of the order of $\tau$, the two interferograms coexist in the cavity and their beats or moire display the differential changes which have occurred. A quantitative measure of these changes is contained in the cross term $\cos \left(\phi_{2}-\phi_{1}\right)$ of the interferogram. Note that the phase $\epsilon$ which contains the phase of pump beams cancels out in the cross term.

\section{Experimental Results}

The actual setup used is shown in Fig. 2. The PCM is an externally pumped single crystal of $\mathrm{BaTiO}_{3}$. The gain of the PCM could be changed by varying the relative intensity of the pumps. The input wavefront after crossing the test area was focused on the PCM with a 16-cm focal length lens. The output was extracted by a pellicle beam splitter (PBS).

To demonstrate the technique, a simple converging spherical beam was first used as an input. The steadystate interferogram (Fig. 3a) shows the expected New- 

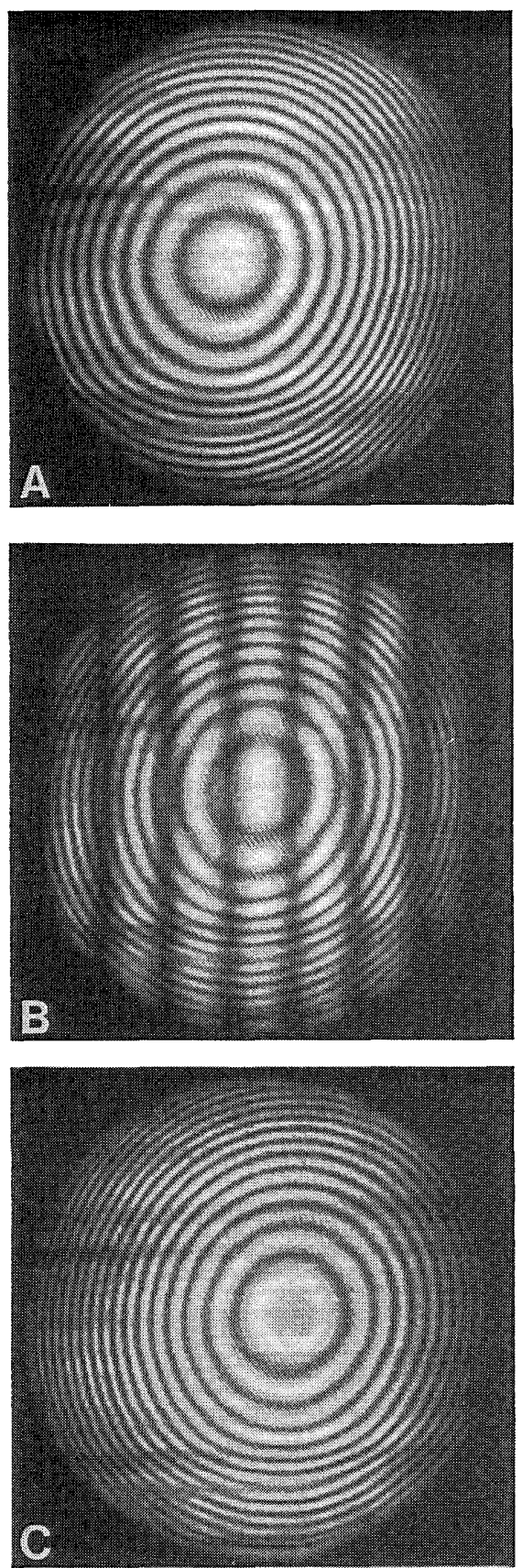

Fig. 3. (A) Initial interferogram of a spherical wavefront. (B) Momentary moiré between inital and final interferogram when the incident wavefront is suddenly translated. (C) Final interferogram of the translated spherical wavefront.

ton's rings due to the interference of two spherical waves of opposite curvatures. When the collimating lens of the beam expander $\mathrm{BE}$ is translated laterally, a new displaced interferogram builds up in the cavity while the old one decays. The moire between these two interferograms (Fig. 3b) appears at the output. It lasts for a couple of seconds (the response time of the PCM for the input irradiance used). After that time, the old interferogram has decayed and only the new one remains (Fig. 3c). A sudden axial translation of the same collimating lens changes the curvature of the
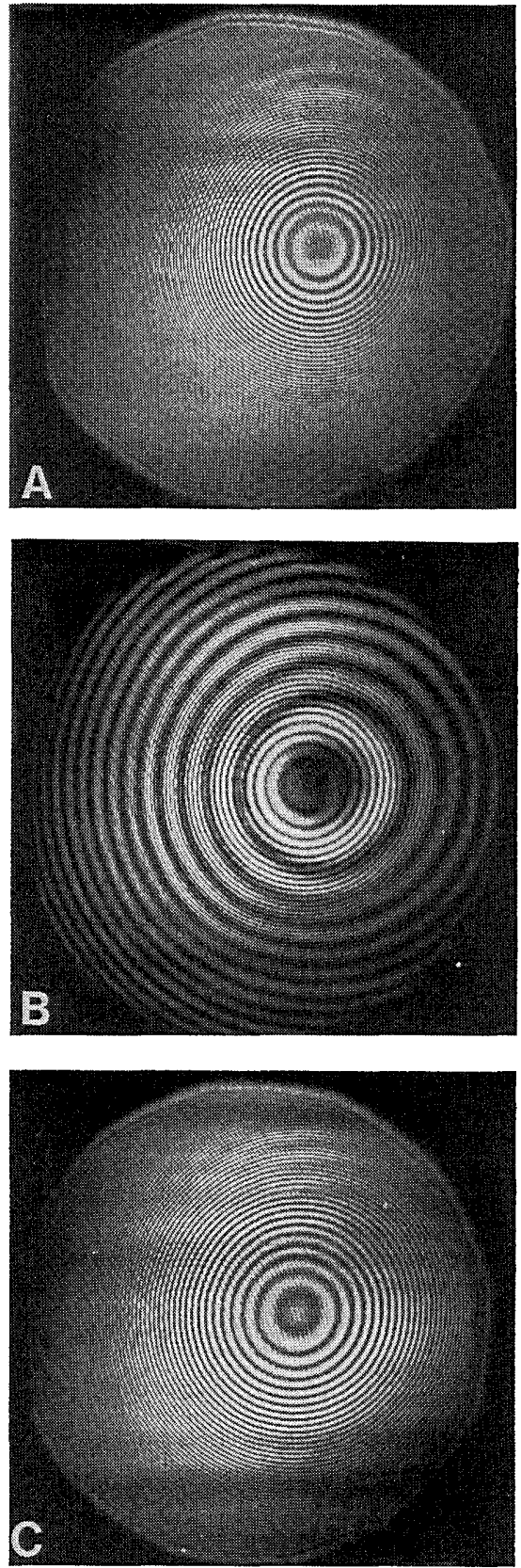

Fig. 4. (A) Initial interferogram of spherical wavefront. (B) Momentary moiré after a sudden change of the curvature of the incident wavefront. (C) Final interferogram.

input wavefront, producing momentarially the moire shown in Fig. 4.

In the previous experiment, the input beam was coaxial with the cavity. Reference fringes can however be easily superposed to the interferogram by tilting the input mirror of the Fabry-Perot. The interferogram of an input plane wave then shows straight fringes (Fig. 5a). After introducing a phase disturbance in the test area (in this case the transparant cover of a plastic box), the moire of Fig. 5(b) appears. After a few seconds, the moire disappears and only the 

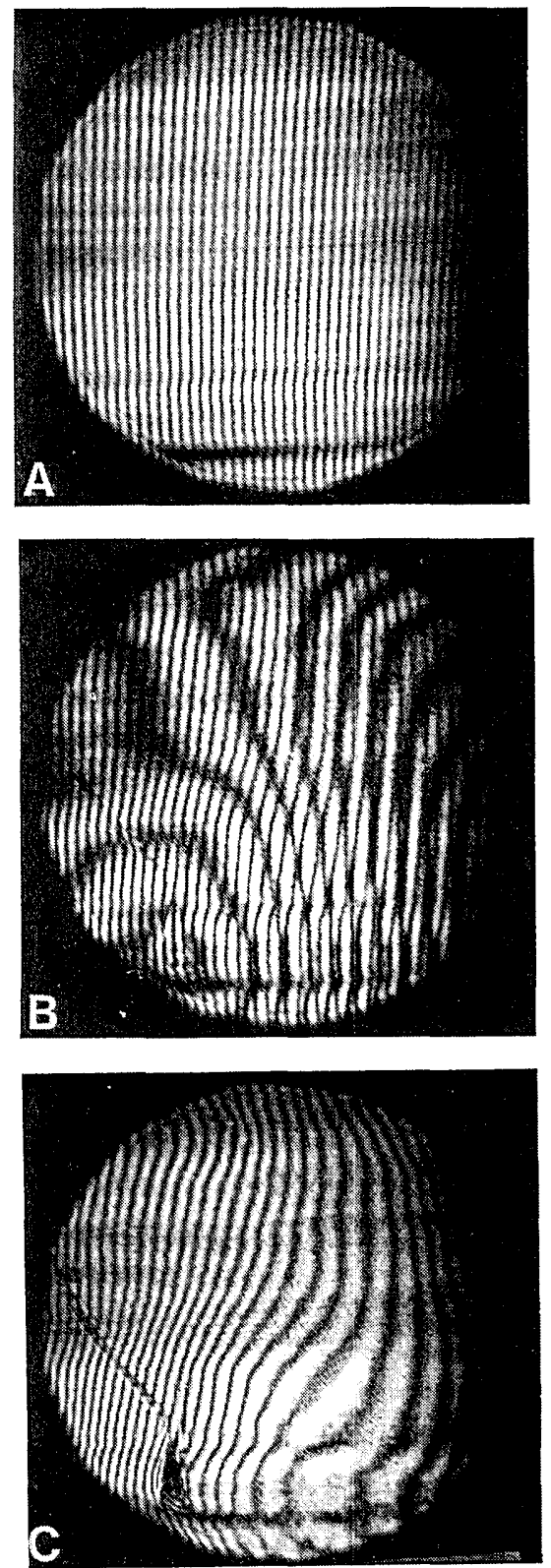

Fig. 5. (A) Initial interferogram of a plane wave with added background fringes. (B) Momentary moiré after the sudden introduction of a transplant plastic box in the test area. (C) Steady-state interferogram of the stationary box.

steady-state self-referencing interferogram of the perturbation remains (Fig. 5c).

These experiments were performed with the resonator below threshold. Above but close to threshold, a similar behavior was observed but occuring on a much longer time scale. In this case, the initial input writes a hologram in the PCM which forces the resonator to oscillate in a spatial mode consisting of the superposition of the input and its complex conjugate (the initial interferogram). After a sudden change, the new input will write a new hologram, forcing the resonator to accept this new input as the most favorable eigenmode. This occurs very slowly however, because the previous pattern can remain in resonance in the cavity for quite a long time. In fact, our assumption that the resonator filling time is small compared with the PCM response time may very well be invalidated. Further above threshold, the output degrades in time, mainly because noisy gratings in the PCM may have a higher gain than the grating imposed by the input and thus contribute noise to the oscillating mode.

\section{Conclusion}

We have described a self-referencing interferometer based on a Fabry-Perot cavity with a phase conjugate mirror rather than on the more commonly used Michelson geometry. This offers several advantages. The cavity generates the wavefront and its complex conjugate colinearly, easing the alignment of the device. Reference fringes can be introduced easily by tilting the input mirror of the cavity. The gain of the PCM can be varied to change the dynamic response of the interferometer. For the experiments, we used a single crystal of $\mathrm{BaTiO}_{3}$ for the PCM. The slow response of this material makes it convenient to display the moire between old and new interferograms after a sudden change of the input wavefront has occured. The interferometer can thus measure differential changes as well as steady-state wavefronts.

Acknowledgement is made to the Thomas F. and Kate Miller Jeffress Memorial Trust for the support of this research.

\section{References}

1. Y. Fainman, E. Lenz, and J. Shamir, "Contouring by Phase Conjugation," Appl. Opt. 20, 158-163 (1981).

2. F. A. Hopf, "Interferometry Using Conjugate-wave Generation," J. Opt. Soc. Am,. 70, 1320-1323 (1980).

3. I. Bar-Joseph, A. Hardy, Y. Katzir, and Y. Silberberg, "Lowpower Phase-Conjugate Interferometry," Opt. Lett. 6, 414-416 (1981).

4. O. Ikeda, T. Suzuki, and T. Sako, "High-accuracy Surface Profile Measuring System Using a BSO Phase Conjugating Mirror," Appl. Opt. 21, 4468-4472 (1982).

5. J. Feinberg, "Interferometer with a Self-pumped Phase-conjugating Mirror," Opt. Lett. 8, 569-571 (1983).

6. D. Z. Anderson, D. M. Lininger, and J. Feinberg, "Optical Tracking Novelty Filter," Opt. Lett. 12, 123-125 (1987).

7. S. Chang, M. Isono, and T. Sako, "Dynamic Interferometry Using Two Phase Conjugate Waves," Appl. Opt. 27, 4735-4738 (1988).

8. N. Tan-no, K. Kawauchi, and K. Yokoto, "Self-interferometry by Dual-wave-front Generation in Degerate Four-wave Mixing," J. Opt. Soc. Am. B 3, 60-64 (1986).

9. A. E. Siegman, P. A. Belanger, and A. Hardy, Ch. 13 "Optical Resonators Using Phase-conjugate Mirrors," in Optical Phase Conjugation, R. A. Fisher, Ed. (Academic, New York, 1983).

10. G. S. Agarwal, A. T. Friberg, and E. Wolf, "Elimination of Distorsion by Phase Conjugation Without Losses or Gains," Opt. Commun. 43, 446-450 (1982).

11. I. Lindsay and J. C. Dainty, "Partial Cancellation of Specular Reflection in the Presence of a Phase Conjugate Mirror," Opt. Commun. 59, 405-410 (1986).

12. A. T. Friberg, M. Kauranen, and R. Salomaa, "Dynamics of Fabry-Perot Resonators with a Phase-conjugate Mirror," J. Opt. Soc. Am. B 3, 1656-1672 (1986). 